\title{
HRTEM Studies of Phase Transformation in Boron Nitride Under High Pressure And High Temperature
}

\author{
J.Y. Huang \\ Department of Physics, Boston College, Chestnut Hill, MA 02467
}

Hexagonal boron nitride (h-BN) transforms to wurzite $\mathrm{BN}$ (w-BN) by a puckering mechanism, in which the boron and nitrogen atoms in a hexagonal-linked layer in h-BN splits along two opposite directions into a boron and a nitrogen double-layer in w-BN [1-3]. The transformation mechanism from w-BN to cubic BN (c$\mathrm{BN}$ ) is unclear. Although the orientation relationships (ORs) among the h-, w-, and c-BN phases are wellknown, the atomic structures of the interfaces, which can not only verify the puckering mechanism but also provide new insight into the phase transformation mechanisms, were rarely reported.

Figure 1 is an interface between $\mathrm{h}-\mathrm{BN}$ and $\mathrm{w}-\mathrm{BN}$ in an h-BN sample after high pressure and high temperature (HPHT) treatment at $7.7 \mathrm{GPa}$ and $1350^{\circ} \mathrm{C}$. The h-BN and w-BN follow the following ORs: $[11 \overline{2} 0]_{\mathrm{h}} / /[11 \overline{2} 0]_{\mathrm{w}}$ and $(0002)_{\mathrm{h}}$ is inclined $0-5^{\circ}$ to $(0002)_{\mathrm{w}}$. The interface contains many steps, as marked by arrowheads; $\mathrm{w}-\mathrm{BN}$ grows via the formation of number of steps at its (0002) plane. The interface planes are either $(1 \overline{1} 00)_{\mathrm{h}}$ or $(0002)_{\mathrm{w}}$. To accommodate the mismatch along the c-axises $\left(\mathrm{c}_{\mathrm{h}}=6.66 \AA\right.$ and $\left.\mathrm{c}_{\mathrm{w}}=4.23 \AA\right),(0002)_{\mathrm{h}}$ is inclined to $(0002)_{\mathrm{w}}$. In many cases $(0002)_{\mathrm{h}}$ transforms continuously to $(0002)_{\mathrm{w}}$, which is a direct evidence of the puckering mechanism [1-3]. More remarkably, strips of c-BN phase appears initially from the h-BN phase, which can not be explained by the puckering mechanism, since direct puckering of h-BN yields w-BN. Diffusion or lattice disruption should be involved during the transformation process.

At higher temperatures, there is a direct hexagonal-to-cubic transformation, and w-BN is not present at the interface. Figs. 2-4 depict three high resolution transmission electron microscopy (HRTEM) images obtained from an h-BN sample after HPHT treatment at $7.7 \mathrm{GPa}$ and $1650^{\circ} \mathrm{C}$, showing interfaces between h-BN and cBN. The interface in Fig. 2 is curved and inclined to both $(0002)_{\mathrm{h}}$ and $(1 \overline{1} 00)_{\mathrm{h}}$. The structure of c-BN is perfect, while that of h-BN near the interface is distorted. The interface in Fig. 3a contains one step; the interface plane is $(0002)_{\mathrm{h}} /(111)_{\mathrm{c}}$. The $(0002)_{\mathrm{h}}$ is slightly tilted to the interface by introducing some half Frank loops. The stacking sequence of h-BN is changed in the phase front of c-BN, as outlined by black lines in Fig. 3a. From a magnified image (Fig.3b), it is seen that the stacking sequence of h-BN is changed to abab..., which is exactly the same stacking sequence as that of graphite. Based on Fig. 3b, we propose a graphite-like $\mathrm{BN}$ structure (hereafter referred to as g-BN) as shown in Fig. 3c. Based on the structural model, the simulated image is inserted in Fig. 3b. There is a good match between the simulated image and the experimental one. In Fig. 4, an amorphous layer appears at the $\mathrm{h}-\mathrm{BN} / \mathrm{c}-\mathrm{BN}$ interface, inferring that the phase transformation is diffusion dominated.

The phase transformation mechanism from $\mathrm{w}-\mathrm{BN}$ to c-BN is still unclear. The $\mathrm{w}-\mathrm{BN} / \mathrm{c}-\mathrm{BN}$ interface shown in Fig. 5 was obtained from an h-BN sample after HPHT treatment of $7.7 \mathrm{GPa}, 1450^{\circ} \mathrm{C}$, and it is seen that strips of twinned c-BN nucleate and grow from w-BN. The interface planes are $(0002)_{\mathrm{w}}$ or $(111)_{\mathrm{c}},(1 \overline{1} 00)_{\mathrm{w}}(\mathrm{left}$ arrowheads) and inclined prismatic planes of w-BN (right arrowheads). However, the growth of c-BN phase appears to be along an inclined prismatic plane of w-BN or $(1 \overline{1} 00)_{\mathrm{w}}$, not the $(0002)_{\mathrm{w}}$ or $(111)_{\mathrm{c}}$.

In summary, the interface structures among $\mathrm{h}-\mathrm{w}$ - and c-BN show that the phase transformation can proceed by different routes, such as: $h \rightarrow w \rightarrow c$ (Fig. 1), $h \rightarrow g \rightarrow c$ (Fig. 3), $h \rightarrow a m \rightarrow c$ (Fig. 4) and $h \rightarrow c$ (Fig. 2). Despite the different phase transformation routes, they all follow the same ORs. The phase transformations also follow a general rule, i.e. at lower temperatures, the formation of $\mathrm{c}-\mathrm{BN}$ always proceeds by the formation of intermediate phases such as $\mathrm{w}-\mathrm{BN}$, and the transformation is martensitic dominated; at higher temperatures, there is a direct h-BN to c-BN transformation, and the transformation is diffusional dominated. The reason for this is that a higher temperature favors atomic diffusion, while at lower temperatures, diffusion is difficult.

\section{References}

[1] J.Y. Huang, and Y.T. Zhu, Defects and Diffusion Forum, Vols.186-187 (2000) 1.

[2] A.V. Kurdyumov, V.F.Britun, and I.A.Petrusha, Diamond Related Mater. 5 (1996) 1225.

[3] J.Y. Huang, and Y.T. Zhu, Chem. Mater. 14 (2002) 1873. 


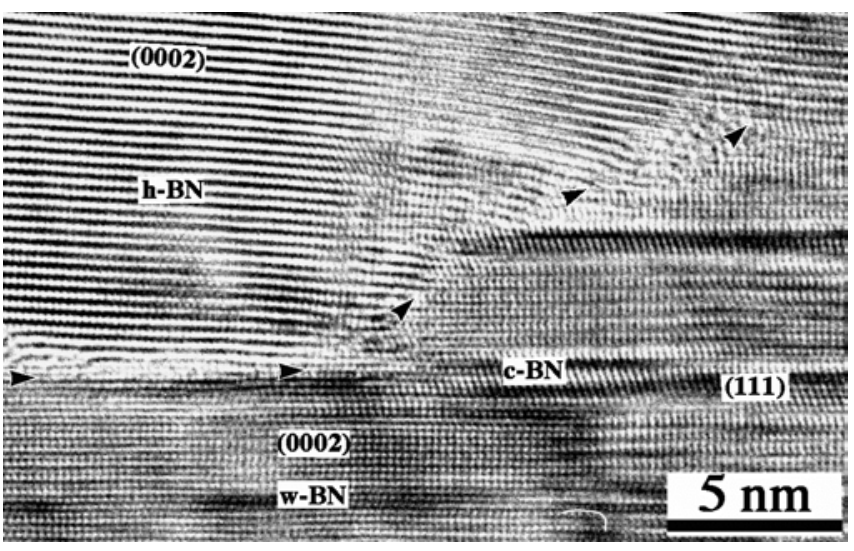

FIG. 1 An interface between h-BN, w-BN and c-BN treated at $7.7 \mathrm{GPa}$ and $1350^{\circ} \mathrm{C}$. They follow definite OR, i.e. $(0002)_{\mathrm{h}} / /(0002)_{\mathrm{w}} / /(111)_{\mathrm{c}},[11 \overline{2} 0]_{\mathrm{h}} / /[11 \overline{2} 0]_{\mathrm{w}}$ $/ /[1 \overline{1} 0]_{\mathrm{c}}$. The interface as marked by white-edged black arrowheads contains many steps.

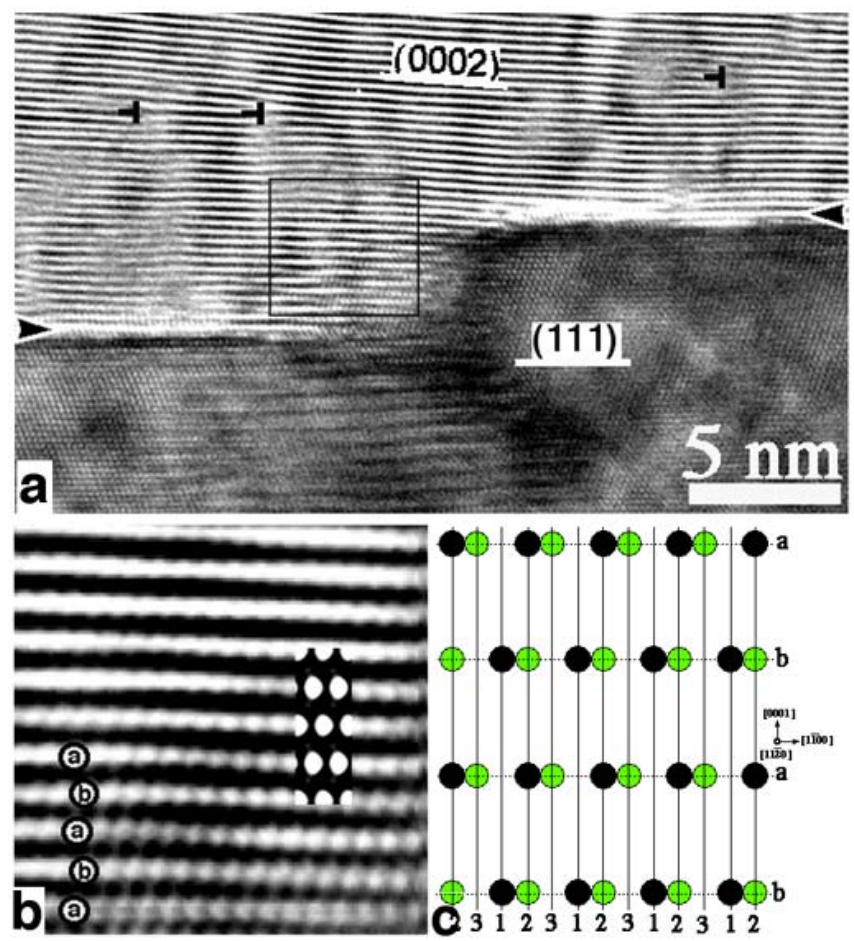

FIG. 3 (a) An interface between h- and c-BN treated at $7.7 \mathrm{GPa}$ and $1650^{\circ} \mathrm{C}$. The $(0002)_{\mathrm{h}}$ is tilted to $(111)_{\mathrm{c}}$ by introducing half Frank loops as marked by some $90^{\circ}$ rotated Ts. (b) An magnified image from the outlined region in (a), showing the change of stacking sequence. Simulated image based on the structural model in (c) was also inserted. (c) A structural model of $\mathrm{g}-\mathrm{BN}$.

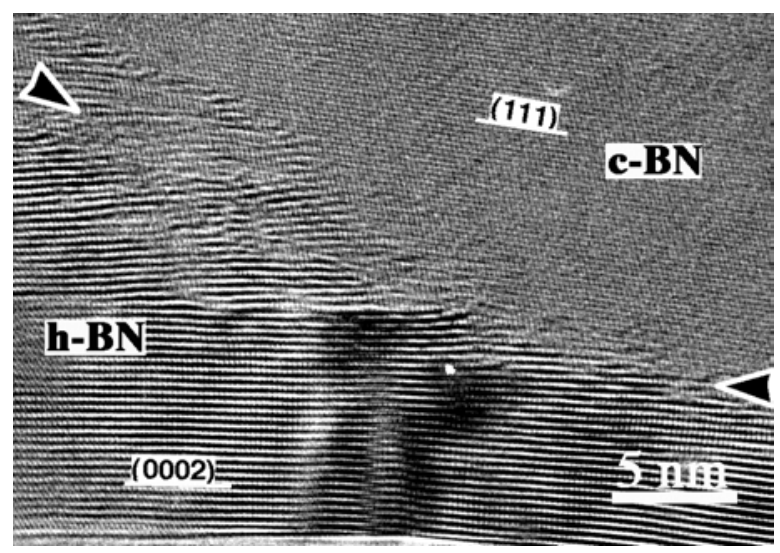

FIG. 2 An interface between h-BN and c-BN treated at $7.7 \mathrm{GPa}, 1650^{\circ} \mathrm{C}$. They follow the same OR as shown in Fig. 1. Remarkably, there is a direct hexagonal to cubic transformation.
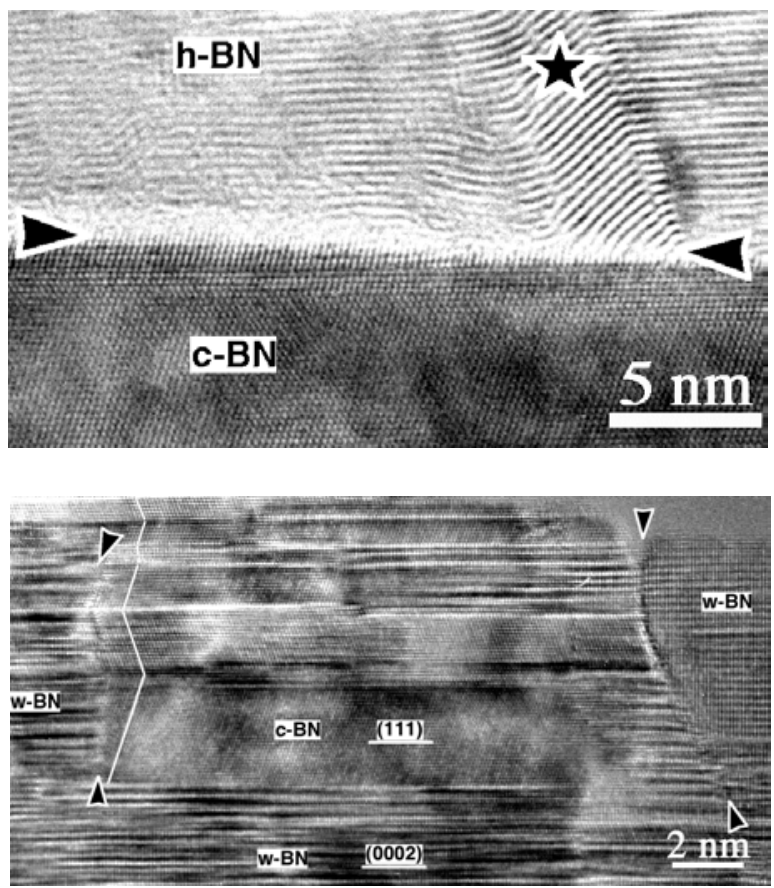

FIG. 4 (Top) An interface between h-BN and c$\mathrm{BN}$ treated at $7.7 \mathrm{GPa}$ and $1650^{\circ} \mathrm{C}$. Note that an amorphous layer exists at the interface.

FIG. 5 (Bottom) A w-BN and c-BN interface treated at $7.7 \mathrm{GPa}$ and $1450^{\circ} \mathrm{C}$. Strips of twinned $\mathrm{c}-\mathrm{BN}$ nucleate and grow from $\mathrm{w}-\mathrm{BN}$, and the growth plane is $(1 \overline{101})_{\mathrm{w}}$ (left arrowheads) or an inclined prismatic plane of w-BN (right arrowheads). 